\title{
Coherent State Quantization and Moment Problem
}

\author{
J. P. Gazeau, M. C. Baldiotti, D. M. Gitman
}

\begin{abstract}
Berezin-Klauder-Toeplitz ("anti-Wick") or "coherent state" quantization of the complex plane, viewed as the phase space of a particle moving on the line, is derived from the resolution of the unity provided by the standard (or gaussian) coherent states. The construction of these states and their attractive properties are essentially based on the energy spectrum of the harmonic oscillator, that is on natural numbers. We follow in this work the same path by considering sequences of non-negative numbers and their associated "non-linear" coherent states. We illustrate our approach with the 2-d motion of a charged particle in a uniform magnetic field. By solving the involved Stieltjes moment problem we construct a family of coherent states for this model. We then proceed with the corresponding coherent state quantization and we show that this procedure takes into account the circle topology of the classical motion.
\end{abstract}

\section{Introduction}

One of the most interesting properties of standard or Glauber coherent states $|z\rangle[1,2,3,4]$ is the Bayesian duality $[5,6]$ that they encode between the discrete Poisson probability distribution, $n \mapsto e^{-|z|^{2}}|z|^{2} / n$ !, of obtaining $n$ quantum excitations ("photons" or "quanta") in a measurement through some counting device, and the continuous Gamma probability distribution measure $|z|^{2} \mapsto e^{-|z|^{2}}|z|^{2} / n$ ! on the classical phase space. For this latter distribution, $|z|^{2}$ is itself a random variable, denoting the average number of photons, given that $n$ photons have been counted. Such a duality underlies the construction of all types of coherent state families, provided they satisfy a resolution of the unity condition. It turns out that this condition is equivalent to setting up a "positive operator valued measure" (POVM) [7, 4] on the phase space. Such a measure, in turn, leads to the quantization of the classical phase space, which associates to each point $z \equiv(q+i p) / \sqrt{2}$ the one dimensional projection operator $\mathrm{P}_{z}$, projecting onto to the subspace generated by the coherent state vector, and then for $z \neq z^{\prime}$, $\left.\mathrm{P}_{z} \mathrm{P}_{z^{\prime}} \neq \mathrm{P}_{z^{\prime}} \mathrm{P}_{z}\right)$. This "Berezin-Klauder-Töplitz" quantization (or "anti-Wick") [1, 8, 9] turns out, in this case, to be equivalent to the canonical quantization procedure. Clearly, this non-commutative version of the complex plane is intrinsically based on the nonnegative integers (appearing in the $n$ ! term). We then follow a similar path by considering sequences of nonnegative numbers which are far or not from the natural numbers [10]. The resulting quantizations will then be looked upon as generalizations of the one yielded by the standard coherent states.

We illustrate our approach with the elementary model of the 2-d motion of a charged particle in a uniform magnetic field $[11,12]$. By using a solution to a version of the Stieltjes moment problem [13, 14] we construct a family of coherent states for this model.
We prove that these states form an overcomplete set that is normalized and resolves the unity. We then carry out the corresponding coherent state quantization and we examine the consequences in terms of its probabilistic, functional, and localization aspects.

This article is organized as follows. In Section 2, we briefly review the standard coherent states and the way they allow painless quantization of the complex plane viewed as a phase space. The so-called nonlinear coherent states built from arbitrary sequences of numbers are described in Section 3 and we show how the moment problem immediately emerges from the exigence of unity resolution. If the positive case, the corresponding quantization of the complex plane is described in Section 4. In Section 5 we apply our formalism to the motion of a charged particle in a uniform magnetic field. There exist two families of coherent states for such a model, namely the Malkin-Man'ko states [15], which are just tensor products of standard coherent states, and the Kowalski-Rembielinski states [16]. By introducing a kind of squeezing parameter $q=e^{\lambda}>1$ we extend the definition of the latter and solve the corresponding Stieltjes moment problem. This allows us to proceed with the quantization of the physical quantities and illustrate our study with numerical investigation.

\section{Quantization with standard coherent states and}

\section{A short review of standard CS}

Let $\mathcal{H}$ be a separable (complex) Hilbert space with orthonormal basis $e_{0}, e_{1}, \ldots, e_{n} \equiv\left|e_{n}\right\rangle, \ldots$. To each complex number $z \in \mathbb{C}$ there corresponds the following vector in $\mathcal{H}$ :

$$
|z\rangle=\sum_{n=0}^{\infty} e^{-\frac{|z|^{2}}{2}} \frac{z^{n}}{\sqrt{n !}}\left|e_{n}\right\rangle
$$

Selected Topics in Mathematical and Particle Physics, Prague, May 5-7, 2009 
Such vectors are the well-known Glauber-KlauderSchrödinger-Sudarshan coherent states or standard coherent states. They are distinguished by many properties. Here we particularly retain the following.

(i) $\langle z \mid z\rangle=1$ (normalization).

(ii) The map $\mathbb{C} \ni z \mapsto|z\rangle$ is continuous (continuity).

(iii) The map $\mathbb{N} \in n \mapsto\left|\left\langle e_{n} \mid z\right\rangle\right|^{2}=e^{-|z|^{2}}|z|^{2 n} / n$ ! is a Poisson probability distribution with average number of occurrences equal to $|z|^{2}$ (discrete probabilistic content).

(iv) The map $\mathbb{C} \ni z \mapsto\left|\left\langle e_{n} \mid z\right\rangle\right|^{2}=e^{-|z|^{2}}|z|^{2 n} / n$ ! is a Gamma probability distribution (with respect to the square of the radial variable) with $n$ as a shape parameter (continuous probabilistic content).

(v) There holds resolution of the unity in $\mathcal{H}$ :

$$
\mathbb{I}=\int_{\mathbb{C}} \frac{\mathrm{d}^{2} z}{\pi} \mathrm{P}_{z}
$$

where $\mathrm{P}_{z}=|z\rangle\langle z|$ is the orthogonal projector on vector $|z\rangle$ and the integral should be understood in the weak sense. The proof is straightforward and stems from the orthogonality of the Fourier exponentials and from the integral expression of the gamma function which solves the moment problem for the factorial $n$ !

$$
\begin{aligned}
\int_{\mathbb{C}} \frac{\mathrm{d}^{2} z}{\pi} \mathrm{P}_{z}= & \sum_{n, n^{\prime}=0}^{\infty}\left|e_{n}\right\rangle\left\langle e_{n^{\prime}}\right| \frac{1}{\sqrt{n ! n^{\prime} !}} . \\
& \int_{\mathbb{C}} \frac{\mathrm{d}^{2} z}{\pi} e^{-|z|^{2}} z^{n} \bar{z}^{n^{\prime}}= \\
& \sum_{n=0}^{\infty}\left|e_{n}\right\rangle\left\langle e_{n^{\prime}}\right|=\mathbb{I} .
\end{aligned}
$$

\section{Berezin-Klauder-Toeplitz- "anti-Wick" quantization or "coherent state quantization"}

Property (v) allows to define

1. a normalized positive operator-valued measure (POVM) on the complex plane equipped with its Lebesgue measure $\frac{\mathrm{d}^{2} z}{\pi}$ and its $\sigma$-algebra $\mathcal{F}$ of Borel sets:

$$
\mathcal{F} \ni \Delta \mapsto \int_{\Delta} \frac{\mathrm{d}^{2} z}{\pi} \mathrm{P}_{z} \in \mathcal{L}(\mathcal{H})^{+},
$$

where $\mathcal{L}(\mathcal{H})^{+}$is the cone of positive bounded operators on $\mathcal{H}$.

2. a quantization of the complex plane, which means that to a function $f(z, \bar{z})$ in the complex plane there corresponds the operator $A_{f}$ in $\mathcal{H}$ defined by

$$
f \mapsto A_{f}=\int_{\mathbb{C}} \frac{\mathrm{d}^{2} z}{\pi} f(z, \bar{z}) \mathrm{P}_{z}=
$$

$$
\begin{gathered}
\sum_{n, n^{\prime}=0}^{\infty}\left|e_{n}\right\rangle\left\langle e_{n^{\prime}}\right| \frac{1}{\sqrt{n ! n^{\prime} !}} \cdot \\
\int_{\mathbb{C}} \frac{\mathrm{d}^{2} z}{\pi} f(z, \bar{z}) e^{-|z|^{2}} z^{n} \bar{z}^{n^{\prime}}
\end{gathered}
$$

provided that weak convergence holds.

For the simplest functions $f(z)=z$ and $f(z)=\bar{z}$ we obtain

$$
\begin{aligned}
A_{z}= & \hat{a}, \quad \hat{a}\left|e_{n}\right\rangle=\sqrt{n}\left|e_{n-1}\right\rangle \\
\hat{a}\left|e_{0}\right\rangle= & 0, \quad(\text { lowering operator) } \\
A_{\bar{z}}= & \hat{a}^{\dagger}, \quad \hat{a}^{\dagger}\left|e_{n}\right\rangle=\sqrt{n+1}\left|e_{n+1}\right\rangle \\
& \text { (raising operator) } .
\end{aligned}
$$

These two basic operators obey the canonical commutation rule : $\left[\hat{a}, \hat{a}^{\dagger}\right]=\mathbb{I}$. The number operator $\hat{N}=\hat{a}^{\dagger} \hat{a}$ is such that its spectrum is exactly $\mathbb{N}$ with eigenvectors $e_{n}: \hat{N}\left|e_{n}\right\rangle=n\left|e_{n}\right\rangle$. The fact that the complex plane has become non-commutative is apparent from the quantization of the real and imaginary parts of $z=\frac{1}{\sqrt{2}}(q+i p)$ :

$$
\begin{aligned}
& A_{q} \stackrel{\text { def }}{=} Q=\frac{1}{\sqrt{2}}\left(\hat{a}+\hat{a}^{\dagger}\right), \\
& A_{p} \stackrel{\text { def }}{=} P=\frac{1}{\sqrt{2} i}\left(\hat{a}-\hat{a}^{\dagger}\right), \quad[Q, P]=i \mathbb{I} .
\end{aligned}
$$

\section{Coherent states for generic sequences}

Let $\mathcal{X}=\left\{x_{n}\right\}_{n \in \mathbb{N}}$ be a strictly increasing sequence such that $x_{0}=0$ and $\lim _{n \rightarrow \infty} x_{n}=\infty$. Then its associated exponential

$$
\mathcal{E}(t)=\sum_{n=0}^{+\infty} \frac{t^{n}}{x_{n} !}, \quad x_{n} ! \equiv x_{1} x_{2} \cdots x_{n}, \quad x_{0} !=1
$$

has an infinite convergence radius. Associated "coherent states" ("non-linear CS" in Quantum Optics) read as elements of $\mathcal{H}$, a separable Hilbert space with orthonormal basis $\left\{\left|e_{n}\right\rangle, n \in \mathbb{N}\right\}$ :

$$
\left|v_{z}\right\rangle=\sum_{n=0}^{\infty} \frac{1}{\sqrt{\mathcal{E}\left(|z|^{2}\right)}} \frac{z^{n}}{\sqrt{x_{n} !}}\left|e_{n}\right\rangle
$$

These vectors still enjoy some properties similar to the standard ones.

(i) $\left\langle v_{z} \mid v_{z}\right\rangle=1$ (normalization).

(ii) The map $\mathbb{C} \ni z \mapsto\left|v_{z}\right\rangle$ is continuous (continuity). 
(iii) The map $\mathbb{N} \in n \mapsto\left|\left\langle e_{n} \mid v_{z}\right\rangle\right|^{2}=\frac{|z|^{2 n}}{\mathcal{E}\left(|z|^{2}\right) x_{n} !}$ is a Poisson-like distribution with average number of occurrences equal to $|z|^{2}$ (discrete probabilistic content).

Consider the discrete probability distribution with parameter $t \geq 0$ :

$$
n \mapsto p(n ; t)=\frac{1}{\mathcal{E}(t)} \frac{t^{n}}{x_{n} !} .
$$

The average of the random variable $n \mapsto x_{n}$ is $\left\langle x_{n}\right\rangle=t$. Contrariwise to the standard case $\mathcal{X}=\mathbb{N}$, the continuous (gammalike) distribution $t \mapsto \frac{1}{\mathcal{E}(t)} \frac{t^{n}}{x_{n} !}$ with parameter $n$ is not a probability distribution with respect to the Lebesgue measure $\mathrm{d} t$ :

$$
\int_{0}^{+\infty} \frac{\mathrm{d} t}{\mathcal{E}(t)} \frac{t^{n}}{x_{n} !} \stackrel{\text { def }}{=} \mu_{n} \neq 1 .
$$

Finding the right measure amounts to solve a usually intractable moment problem. So, the map $\mathbb{C} \ni z \mapsto$ $\left|\left\langle e_{n} \mid v_{z}\right\rangle\right|^{2}=|z|^{2 n} /\left(\mathcal{E}\left(|z|^{2}\right) x_{n} !\right)$ is not a (Gamma-like) probability distribution (with respect to the square of the radial variable in the complex plane) with $x_{n+1}$ as a shape parameter, and this is a serious setback for the Berezin-Toeplitz quantization program. Indeed there is no reason to get now the resolution of the unity: with $\mathrm{P}_{z}=\left|v_{z}\right\rangle\left\langle v_{z}\right|$,

$$
\begin{aligned}
\int_{\mathbb{C}} \frac{\mathrm{d}^{2} z}{\pi} \mathrm{P}_{z}= & \sum_{n, n^{\prime}=0}^{\infty}\left|e_{n}\right\rangle\left\langle\left( e_{n^{\prime}} \mid \frac{1}{\sqrt{x_{n} ! x_{n}^{\prime} !}} .\right.\right. \\
& \int_{\mathbb{C}} \frac{\mathrm{d}^{2} z}{\pi} \frac{1}{\mathcal{E}\left(|z|^{2}\right)} z^{n} \bar{z}^{n^{\prime}}= \\
& \sum_{n=0}^{\infty} \frac{1}{x_{n} !} \mathcal{I}(n)\left|e_{n}\right\rangle\left\langle\left( e_{n} \mid \stackrel{\text { def }}{=} \mathbb{F} .\right.\right.
\end{aligned}
$$

Here $\mathbb{F}$ is a diagonal operator determined by the sequence of integrals $\mathcal{I}(n)=\int_{0}^{+\infty} t^{n} \frac{\mathrm{d} t}{\mathcal{E}(t)}$. These integrals form a sequence of Stieltjes moments for the measure $\frac{\mathrm{d} t}{\mathcal{E}(t)}$.

If the moment problem has a solution. Suppose that the Stieltjes moment problem [13, 14] has a solution for the sequence $\left(x_{n} !\right)_{n \in \mathbb{N}}$, i.e. there exists a probability distribution $t \mapsto w(t)$ on $[0,+\infty)$ with infinite support such that

$$
x_{n} !=\int_{0}^{+\infty} t^{n} w(t) \mathrm{d} t .
$$

We know that a necessary and sufficient condition for this is that the two matrices

$$
\left(\begin{array}{ccccc}
1 & x_{1} ! & x_{2} ! & \ldots & x_{n} ! \\
x_{1} ! & x_{2} ! & x_{3} ! & \ldots & x_{n+1} ! \\
x_{2} ! & x_{3} ! & x_{4} ! & \ldots & x_{n+2} ! \\
\vdots & \vdots & \vdots & \ddots & \vdots \\
x_{n} ! & x_{n+1} ! & x_{n+2} ! & \ldots & x_{2 n} !
\end{array}\right),
$$

$$
\left(\begin{array}{ccccc}
x_{1} ! & x_{2} ! & x_{3} ! & \ldots & x_{n+1} ! \\
x_{2} ! & x_{3} ! & x_{4} ! & \ldots & x_{n+2} ! \\
x_{3} ! & x_{4} ! & x_{5} ! & \ldots & x_{n+3} ! \\
\vdots & \vdots & \vdots & \ddots & \vdots \\
x_{n+1} ! & x_{n+2} ! & x_{n+3} ! & \ldots & x_{2 n+1} !
\end{array}\right)
$$

have strictly positive determinants for all $n$. Then, a natural approach is just to modify the measure on $\mathbb{C}$ by including the weight $w\left(|z|^{2}\right) \mathcal{E}\left(|z|^{2}\right)$. We then obtain the resolution of the identity:

$$
\begin{aligned}
& \int_{\mathbb{C}} \frac{\mathrm{d}^{2} z}{\pi} w\left(|z|^{2}\right) \mathcal{E}\left(|z|^{2}\right) \mathrm{P}_{z}= \\
& \sum_{n, n^{\prime}=0}^{\infty}\left|e_{n}\right\rangle\left\langle\left( e_{n^{\prime}} \mid \frac{1}{\sqrt{x_{n} ! x_{n}^{\prime} !}} \cdot\right.\right. \\
& \int_{\mathbb{C}} \frac{\mathrm{d}^{2} z}{\pi} w\left(|z|^{2}\right) z^{n} \bar{z}^{n^{\prime}}= \\
& \sum_{n=0}^{\infty} \frac{1}{x_{n} !} \int_{0}^{+\infty} t^{n} w(t) \mathrm{d} t\left|e_{n}\right\rangle\left\langle\left( e_{n} \mid=\mathbb{I} .\right.\right.
\end{aligned}
$$

If the moment problem is solved by a measure $t \mapsto$ $w(t)$, then the vectors $\left|v_{z}\right\rangle$ enjoy all needed properties for quantization:

(iv) The map $\mathbb{C} \ni z \mapsto\left|\left\langle e_{n} \mid v_{z}\right\rangle\right|^{2}=|z|^{2 n} /\left(\mathcal{E}\left(|z|^{2}\right) x_{n}\right.$ !) is a (Gamma-like) probability distribution (with respect to the square of the radial variable) with $x_{n+1}$ as a shape parameter and with respect to the modified measure on the complex plane

$$
\nu(d z) \stackrel{\text { def }}{=} w\left(|z|^{2}\right) \mathcal{E}\left(|z|^{2}\right) \frac{\mathrm{d}^{2} z}{\pi} .
$$

Note that we might face with $\left(x_{n} !\right)$ an indeterminate moment sequence, which means that there are several representing measures. Then to each such a measure there corresponds a probability distribution on the classical phase space to be interpreted in terms of statistical mechanics.

If the moment problem has no (explicit) solution. See an alternative in [10].

\section{CS quantization with sequence $\mathcal{X}$}

If the moment problem has an explicit solution, one can proceed with the corresponding CS quantization of the complex plane since the family of vectors $\left|v_{z}\right\rangle$ solves the unity : with $\mathrm{P}_{z}=\left|v_{z}\right\rangle\left\langle v_{z}\right|$,

$$
\begin{aligned}
\int_{\mathbb{C}} \nu(\mathrm{d} z) \mathrm{P}_{z}= & \sum_{n, n^{\prime}=0}^{\infty}\left|e_{n}\right\rangle\left\langle e_{n^{\prime}}\right| \frac{1}{\sqrt{x_{n} ! x_{n^{\prime}} !}} . \\
& \int_{\mathbb{C}} \frac{\mathrm{d}^{2} z}{\pi} w\left(|z|^{2}\right) z^{n} \bar{z}^{n^{\prime}}= \\
& \sum_{n=0}^{\infty} \frac{1}{x_{n} !}\left|e_{n}\right\rangle\left\langle e_{n}\right|=\mathbb{I},
\end{aligned}
$$


We proceed with this quantization like in the standard case: to a function $f(z, \bar{z})$ in the complex plane there corresponds the operator $A_{f}$ in $\mathcal{H}$ defined by

$$
\begin{aligned}
f \mapsto A_{f}= & \int_{\mathbb{C}} \frac{\mathrm{d}^{2} z}{\pi} f(z, \bar{z}) w\left(|z|^{2}\right) \mathcal{E}\left(|z|^{2}\right) \mathrm{P}_{z}= \\
& \sum_{n, n^{\prime}=0}^{\infty}\left|e_{n}\right\rangle\left\langle e_{n^{\prime}}\right| \frac{1}{\sqrt{x_{n} ! x_{n^{\prime}} !}} \cdot \\
& \int_{\mathbb{C}} \frac{\mathrm{d}^{2} z}{\pi} w\left(|z|^{2}\right) f(z, \bar{z}) z^{n} \bar{z}^{n^{\prime}}
\end{aligned}
$$

provided that weak convergence holds. For the simplest functions $f(z, \bar{z})=z$ and $f(z, \bar{z})=\bar{z}$ we obtain

$$
\begin{aligned}
A_{z}= & \hat{a}, \quad \hat{a}\left|e_{n}\right\rangle=\sqrt{x_{n}}\left[e_{n-1}\right\rangle, \\
\hat{a}\left|e_{0}\right\rangle= & 0, \quad \text { (lowering operator) } \\
A_{\bar{z}}= & \hat{a}^{\dagger}, \quad \hat{a}^{\dagger}\left|e_{n}\right\rangle=\sqrt{x_{n+1}}\left|e_{n+1}\right\rangle \\
& \text { (raising operator). }
\end{aligned}
$$

These two basic operators obey the commutation rule : $\left[\hat{a}, \hat{a}^{\dagger}\right]=x_{N+1}-x_{N} \stackrel{\text { def }}{=} \Delta_{N}$. The operator $x_{N}$ is defined by $x_{N}=\hat{a}^{\dagger} \hat{a}$ and is such that its spectrum is exactly the sequence $\mathcal{X}$ with eigenvectors $e_{n}: x_{N}\left|e_{n}\right\rangle=$ $x_{n}\left|e_{n}\right\rangle$. The triple $\left\{\hat{a}, \hat{a}^{\dagger}, \Delta_{N}\right\}$ equipped with the operator commutator $[\cdot, \cdot]$ generates (generically) an infinite Lie algebra which replaces the Weyl-Heisenberg Lie algebra. The quantization of the real and imaginary parts of $z=\frac{1}{\sqrt{2}}(q+i p)$ yields position and momentum operators corresponding to the sequence $\mathcal{X}$,

$$
\begin{aligned}
& A_{q} \stackrel{\text { def }}{=} Q=\frac{1}{\sqrt{2}}\left(\hat{a}+\hat{a}^{\dagger}\right), \\
& A_{p} \stackrel{\text { def }}{=} P=\frac{1}{\sqrt{2} i}\left(\hat{a}-\hat{a}^{\dagger}\right), \quad[Q, P]=i \Delta_{N},
\end{aligned}
$$

together with new quantum localization properties.

\section{An example: charged particle in a magnetic field}

Consider a classical nonrelativistic particle, charge $-e$, moving in the plane $\left(x^{1}, x^{2}\right)$ and interacting with a constant and uniform magnetic field of intensity $B$ perpendicular to the plane, described by a vector potential A only $\left(A^{0}=0\right)$. The Hamiltonian of the particle is $H(\mathbf{x}, \mathbf{p})=\frac{1}{2 \mu}\left[\mathbf{p}+\frac{e}{c} \mathbf{A}(\mathbf{x})\right]^{2}, \mathbf{x}=$ $\left(x^{1}, x^{2}\right), \mathbf{p}=\left(p_{1}, p_{2}\right)$. With the symmetric gauge $\hat{A}_{i}=-\frac{B}{2} \varepsilon_{i j} \hat{x}^{j}, i, j, k=1,2$, the quantum Hamiltonian takes the form $\hat{H}=\frac{1}{2 \mu}\left(\hat{P}_{1}^{2}+\hat{P}_{2}^{2}\right)$. The $\hat{P}_{i}$, $i=1,2$, are components of the kinematic momentum operator,

$$
\hat{P}_{i}=\hat{p}_{i}-\frac{e B}{2 c} \varepsilon_{i j} \hat{x}^{j},\left[\hat{P}_{1}, \hat{P}_{2}\right]=-i \hbar \frac{e B}{c},
$$

where $\varepsilon_{i j}$ is the Levi-Civita symbol.

\section{Kowalski \& Rembielinski coherent states}

Kowalski and Rembielinski [16] have proposed the construction of CS for a particle in a uniform magnetic field by using their coherent states for the circle [17]. The latter are constructed from the angular momentum operator $\hat{J}$ and the unitary operator $\hat{U}$ that represents the position of the particle on the unit circle. These operators obey the commutation relations

$$
[\hat{J}, \hat{U}]=U,\left[\hat{J}, \hat{U}^{+}\right]=-\hat{U}^{+}
$$

The introduction of these coherent states permits to avoid the problem of the infinite degeneracy present in the approach followed by Man'ko and Malkin [15], and, in addition, takes into in account the momentum part of the phase space. Consequently, the so obtained CS offer a better way to compare the quantum behavior of the system with the classical trajectories in the phase space.

Let us introduce the centre-coordinate operators

$$
\hat{x}_{0}^{1}=\hat{x}^{1}-\frac{1}{\mu \omega} \hat{P}_{2}, \quad \hat{x}_{0}^{2}=\hat{x}^{2}+\frac{1}{\mu \omega} \hat{P}_{1},
$$

where $\omega=e B / \mu$ is the cyclotron frequency. The $\hat{x}_{0}^{i}$ are integral of motion, $\left[H, \hat{x}_{0}^{i}\right]=0$. Relative motion coordinate operators,

$$
\begin{aligned}
& \hat{r}^{1}=\hat{x}^{1}-\hat{x}_{0}^{1}=\frac{1}{\mu \omega} \hat{P}_{2}, \\
& \hat{r}^{2}=\hat{x}^{2}-\hat{x}_{0}^{1}=-\frac{1}{\mu \omega} \hat{P}_{1} .
\end{aligned}
$$

Introduce now the operators

$$
\begin{aligned}
\hat{r}_{0 \pm} & =\hat{x}_{0}^{1} \pm i \hat{x}_{0}^{2} \\
\hat{r}_{ \pm} & =\hat{r}^{1} \pm i \hat{r}^{2}=\frac{1}{\mu \omega}\left(\hat{P}_{2} \mp i \hat{P}_{1}\right) .
\end{aligned}
$$

They obey the commutation rules

$$
\begin{aligned}
{\left[\hat{r}_{0+}, \hat{r}_{0-}\right] } & =2 \frac{\hbar}{\mu \omega}, \quad\left[\hat{r}_{+}, \hat{r}_{-}\right]=-2 \frac{\hbar}{\mu \omega} \\
{\left[\hat{r}_{0 \pm}, \hat{r}_{ \pm}\right] } & =0
\end{aligned}
$$

The "relative" angular momentum operator $\hat{J}$ is proportional to the Hamiltonian

$$
\begin{aligned}
& \hat{J}= \hat{r}_{1} \hat{P}_{2}-\hat{r}_{2} \hat{P}_{1}=-\frac{2}{\omega} \hat{H}= \\
& \mu \omega \hat{r}_{+} \hat{r}_{-}+\hbar=\mu \omega \hat{r}_{-} \hat{r}_{+}-\hbar .
\end{aligned}
$$

Due to the rules,

$$
\left[J, \hat{r}_{0 \pm}\right]=0, \quad\left[J, \hat{r}_{ \pm}\right]= \pm 2 \hbar \hat{r}_{ \pm}
$$

$\hat{J}$ can be viewed as the generator of rotations about the axis passing through the classical point $\left(x_{0}^{1}, x_{0}^{2}\right)$ 
and perpendicular to the $\left(x^{1}, x^{2}\right)$ plane. The nonunitary operator $\hat{r}_{-}$describes to a certain extent the angular position of the particle on a circle. The symmetries and the integrability of the model can be encoded into the two independent Weyl-Heisenberg algebras, one for the center of circular orbit and the other for the relative motion. They allow one to construct the Fock space with orthonormal basis $\{|m, n\rangle \equiv|m\rangle \otimes|n\rangle, m, n \in \mathbb{Z}\}$, as repeated actions of the raising operators $\hat{r}_{0-}$ and $\hat{r}_{+}$,

$$
\begin{aligned}
\hat{r}_{0-}|m\rangle & =\sqrt{\frac{2 \hbar(m+1)}{\mu \omega}}|m+1\rangle, \\
\hat{r}_{+}|n\rangle & =\sqrt{\frac{2 \hbar(n+1)}{\mu \omega}}|n+1\rangle . \\
\hat{r}_{0+}|m\rangle & =\sqrt{\frac{2 \hbar m}{\mu \omega}}|m-1\rangle, \\
\hat{r}_{-}|n\rangle & =\sqrt{\frac{2 \hbar n}{\mu \omega}}|n-1\rangle,
\end{aligned}
$$

and the eigenvalue equation

$$
\hat{J}|m, n\rangle=(2 n+1) \hbar|m, n\rangle .
$$

The K\&R CS $\left|z_{0}, \zeta\right\rangle$ are constructed in the Hilbert space spanned by the orthonormal basis as solution to the eigenvalue equation:

$$
\begin{aligned}
\hat{r}_{0+}\left|z_{0}, \zeta\right\rangle & =z_{0}\left|z_{0}, \zeta\right\rangle, \\
\hat{Z}\left|z_{0}, \zeta\right\rangle & =\zeta\left|z_{0}, \zeta\right\rangle, z_{0}, \zeta \in \mathbb{C},
\end{aligned}
$$

where $\hat{Z}=e^{\frac{1}{2}(\hat{J} / \hbar+1)} \hat{r}_{-}$. The projection of these CS in this Fock basis reads as

$$
\left\langle m, n \mid \zeta, z_{0}\right\rangle=\frac{e^{-\frac{\left|\tilde{z}_{0}\right|^{2}}{2}}}{\sqrt{\mathcal{E}\left(|\tilde{\zeta}|^{2}\right)}} \frac{\tilde{z}_{0}^{m}}{\sqrt{m !}} \frac{\tilde{\zeta}^{n}}{\sqrt{n !}} e^{-\frac{1}{2} n(n+1)},
$$

where $\tilde{z}_{0}=\sqrt{\frac{\mu \omega}{2 \hbar}} z_{0}, \quad \tilde{\zeta}=\sqrt{\frac{\mu \omega}{2 \hbar}} \zeta$. The normalization factor involves the function

$$
\mathcal{E}(t)=\sum_{n=0}^{\infty} e^{-n(n+1)} \frac{t^{n}}{n !} \equiv \sum_{n=0}^{\infty} \frac{t^{n}}{x_{n} !}
$$

where we recognize a generalized exponential with $x_{n} \equiv e^{2 n} n$.

\section{Squeezing/deforming the $K \& \mathbf{R}$ states}

The introduction of a "squeezing" parameter $\lambda$ allows us to generalize the previous CS of a charged particle in a uniform magnetic field as an eigenvector of the commuting operators $\hat{r}_{0+}$ and $\hat{Z}_{\lambda}$,

$$
\begin{aligned}
\hat{r}_{0+}\left|z_{0}, \zeta\right\rangle & =z_{0}\left|z_{0}, \zeta\right\rangle, \hat{Z}_{\lambda}\left|z_{0}, \zeta\right\rangle=\zeta\left|z_{0}, \zeta\right\rangle, \\
\hat{Z}_{\lambda} & =\exp \left[\frac{\lambda}{4}(\hat{J} / \hbar+1)\right] \hat{r}_{-} .
\end{aligned}
$$

Operator $\hat{Z}_{\lambda}$ coincides with the $\mathrm{K} \& \mathrm{R} \hat{Z}$ for $\lambda=2$, and with just $\hat{r}_{-}$for $\lambda=0$, i.e., the case of Malkin-Man'ko CS, which are actually tensor products of standard CS. Operator $\hat{Z}_{\lambda}$ controls the dispersion relations of the angular momentum $\hat{J}$ and of the "position operator" $\hat{r}_{-}$. The corresponding CS read:

$$
\left|z_{0}, \zeta\right\rangle=\frac{e^{-\frac{\left|\tilde{z}_{0}\right|^{2}}{2}}}{\sqrt{\mathcal{E}_{\lambda}\left(|\tilde{\zeta}|^{2}\right)}} \sum_{m, n} \frac{\tilde{z}_{0}^{m}}{\sqrt{m !}} \frac{\tilde{\zeta}^{n}}{\sqrt{x_{n} !}}|m, n\rangle
$$

with $\mathcal{E}_{\lambda}(t)=\sum_{n=0}^{\infty} t^{n} / x_{n}$ ! and $x_{n} \equiv e^{n \lambda} n$. The complex numbers $z_{0}$ and $\zeta$ parameterize, respectively, the position of the centre of the circle and the classical phase space state of the circular motion. Some properties of these CS make them more suitable with regard to the semi-classical behavior of a charged particle in a magnetic field, in comparison with the Malkin-Man'ko CS. The generalization involving $\lambda$ allows one to exhibit better these interesting characteristics.

\section{Resolution of the moment problem}

The $\lambda$-CS $\left|z_{0}, \zeta\right\rangle$ are the tensor product of the states $\left|z_{0}\right\rangle$ and $|\zeta\rangle$, where the first one is a standard CS. So, in order to perform the CS quantization, we concentrate only on the states $|\zeta\rangle$. For convenience, we put $\mu \omega / 2 \hbar=1$, and so $\tilde{\zeta}=\zeta$. Then, in the Fock basis $\{|n\rangle\}$,

$$
\begin{aligned}
|\zeta\rangle & =\frac{1}{\sqrt{\left.\mathcal{E}_{\lambda}|\zeta|^{2}\right)}} \sum_{n=0}^{+\infty} \frac{\zeta^{n}}{\sqrt{x_{n} !}}|n\rangle, \\
\mathcal{E}_{\lambda}(t) & =\sum_{n=0}^{+\infty} \frac{t}{x_{n} !}, \quad x_{n}=e^{\lambda n} n .
\end{aligned}
$$

They resolve the unity in the Fock space spanned by the kets $|n\rangle$,

$$
\int_{\mathbb{C}} \varpi_{\lambda}\left(|\zeta|^{2}\right) \frac{d^{2} \zeta}{\pi}|\zeta\rangle\langle\zeta|=I .
$$

The weight function $\varpi_{\lambda}$ solves the moment problem

$$
\begin{aligned}
\int_{0}^{\infty} t^{n} \varpi_{\lambda}(t) \mathrm{d} t & =n ! \exp \left\{\frac{\lambda n(n+1)}{2}\right\} \equiv x_{n} ! \\
\lambda \geq 0 &
\end{aligned}
$$

and is given under the form of the Laplace transform,

$$
\begin{aligned}
\varpi_{\lambda}(t)= & \frac{e^{-\lambda / 2}}{\sqrt{2 \pi \lambda}} \int_{0}^{+\infty} \mathrm{d} u \exp \left(-e^{-\lambda / 2} t u\right) e^{-\frac{(\ln u)^{2}}{2 \lambda}}= \\
& \frac{e^{-\lambda / 2}}{\sqrt{2 \pi \lambda}} \mathcal{L}\left[e^{-\frac{(\ln u)^{2}}{2 \lambda}}\right]\left(e^{-\lambda / 2} t\right) .
\end{aligned}
$$




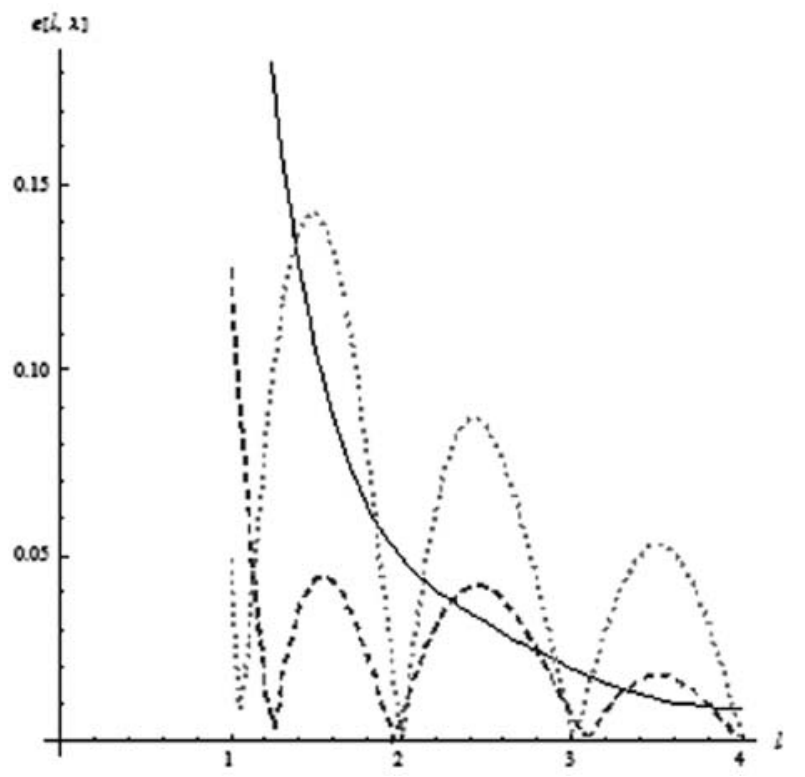

Fig. 1: Error function as a function of $l$ for $\lambda=2$ (solid line), $\lambda=4$ (dashed line) and $\lambda=6$ (dotted line). We see that, with the $\lambda$-CS, this approximation can be improved, for $|l| \leq 1$, by increasing the value of $\lambda$

\section{CS quantization}

The corresponding CS quantization of functions on the complex plane is the map

$$
\begin{aligned}
f(\zeta, \bar{\zeta}) \mapsto & \int_{\mathbb{C}} \frac{\mathrm{d}^{2} \zeta}{\pi} \varpi_{\lambda}\left(|\zeta|^{2}\right) \\
& f(\zeta, \bar{\zeta}) \mathcal{E}_{\lambda}\left(|\zeta|^{2}\right)|\zeta\rangle\langle\zeta| \stackrel{\text { def }}{=} \hat{f}
\end{aligned}
$$

As expected, the CS quantization of the variables $\zeta$ and $\bar{\zeta}$ yields

$$
\zeta \mapsto \hat{\zeta}=\hat{Z}_{\lambda}, \quad \bar{\zeta} \mapsto \hat{\zeta}=\hat{Z}_{\lambda}^{\dagger}
$$

\section{Numerical analysis}

One convenient criterion to evaluate the closeness of the introduced $\lambda$-CS to the classical phase space consists in verifying how closely the expectation value of the angular momentum operator approaches the respective classical quantity. This can be implemented through the evaluation of the relative error

$$
e(\lambda, l)=\frac{\left|\left(\langle\hat{J}\rangle_{\zeta} / \hbar-l\right)\right|}{l}
$$

with the expectation value of the angular momentum given by

$$
\begin{aligned}
\langle\hat{J}\rangle_{\zeta} & =\langle\zeta|\hat{J}| \zeta\rangle=\frac{1}{\mathcal{E}_{q}\left(|\zeta|^{2}\right)} \sum_{n=0}^{+\infty}|\zeta|^{2 n} \frac{(2 n+1)}{x_{n} !} \\
x_{n} & =e^{\frac{\lambda}{2} n} n .
\end{aligned}
$$

The parameter $\zeta$ are related with the classical angular momentum $l=\mu \omega r^{2}$ (where $r$ is the classical radius) by

$$
|\zeta|=\sqrt{\frac{l}{\mu \omega}} \exp \left(\frac{\lambda}{4} l\right) .
$$

Kowalski and Rembielinski observed that the approximate equality $\langle\hat{J}\rangle_{\zeta} \simeq l$ does not hold for arbitrary small $l$, being really acceptable for $|l|>1$ only.

\section{References}

[1] Klauder, I. R., Skagerstam, B. S.: Coherent States, Applications in Physics and Mathematical Physics, World Scientific, Singapore, 1985, pp. 991.

[2] Perelomov, A. M.: Generalized Coherent States and Their Applications, Springer-Verlag, New York 1986.

[3] Malkin, I. A., Man'ko, V. I.: Dynamical Symmetries and Coherent States of Quantum Systems, Nauka, Moscow, 1979, pp. 320.

[4] Ali, S. T., Antoine, J. P., Gazeau, J.-P.: Coherent states, wavelets and their generalizations. Graduate Texts in Contemporary Physics, SpringerVerlag, New York, 2000.

[5] Ali, S. T., Gazeau, J.-P., Heller, B.: J. Phys. A: Math. Theor., 41 (2008) 365302.

[6] Gazeau, J.-P.: Coherent States in Quantum Physics, Wiley-VCH, Berlin, 2009.

[7] Holevo, A. S.: Statistical Structure of Quantum Theory, Springer-Verlag, Berlin, 2001.

[8] Berezin, F. A.: Comm. Math. Phys. 40 (1975) 153.

[9] Chakraborty, B., Gazeau, J.-P., Youssef, A.: arXiv:0805.1847v1.

[10] Ali, S. T., Balková, L., Curado, E. M. F., Gazeau, J.-P., Rego-Monteiro, M. A., Rodrigues, Ligia M. C. S., Sekimoto, K.: J. Math. Phys., 50, 043517-1-28 (2009).

[11] Baldiotti, M. C., Gazeau, J.-P., Gitman, D. M.: Phys. Lett. A, 373, 1916-1920 (2009); Erratum: Phys. Lett. A, 373, 2600 (2009).

[12] Baldiotti, M. C., Gazeau, J.-P., Gitman, D. M.: Phys. Lett. A 373, 3 937-3943 (2009).

[13] Stieltjes, T.: Ann. Fac. Sci. Univ. Toulouse 8 (1894-1895), J1-J122; 9, A5-A47.

[14] Simon, B.: Adv. in Math. 137 (1998), 82-203. 
[15] Malkin, I. A., Man'ko, V. I.: Zh. Eksp. Teor. Fiz. 55 (1968) 1014 [Sov. Phys. - JETP 28, no. 3 (1969) 527].

[16] Kowalski, K., Rembielinski, J.: J. Phys. A 38 (2005) 8247.

[17] Kowalski, K., Rembielinsk, J., Papaloucas, L. C.: J. Phys. A 29 (1996) 4149.
J. P. Gazeau

E-mail: gazeau@apc.univ-paris7.fr

Laboratoire APC

Université Paris Diderot Paris 710

rue A. Domon et L. Duquet

75205 Paris Cedex 13, France

M. C. Baldiotti, D. M. Gitman

E-mail: baldiott@fma.if.usp.br, gitman@dfn.if.usp.br Instituto de Física, Universidade de São Paulo

Caixa Postal 66318-CEP

05315-970 São Paulo, S.P., Brazil 